\title{
Bootstrap Current in Enhanced Reversed Shear Tokamaks for Volume Neutron Source Applications
}

\author{
W. A. Houlberg \\ Oak Ridge National Laboratory \\ Oak Ridge, Tennessee 37831-8071
}

\begin{abstract}
The bootstrap current is evaluated for two reference tokamak designs for a Volume Neutron Source (VNS). One is a larger aspect ratio design using superconducting coils (VNS-SC) and the other is a small aspect ratio design using a solid core with normal conducting toroidal field coils (VNS-ST). The target plasma profiles are taken as representative of the recently observed enhanced reverse shear plasmas with hollow magnetic safety factor $(q)$ profiles in the core and correspondingly peaked density profiles. The higher $q$ in the plasma center in combination with peaked density is shown to move the peak in the bootstrap current toward the plasma center. This reduces the current drive requirements to a very small axial seed current and a source localized around the location of the desired minimum in the $q$ profile. Very high bootstrap current fractions can be attained in the VNS-SC design with normalized betas (defined in terms of the vacuum toroidal magnetic field at the geometric center of the plasma) of $\beta_{N} \leq 3.5$. The bootstrap current is lower in the VNSST design because of its lower aspect ratio: the highest bootstrap fraction found in these limited cases is $50 \%$ at $\beta_{N}=5$.
\end{abstract}

\section{INTRODUCTION}

Steady state fusion reactors are attractive because they simplify many engineering design aspects of the power handling and power conversion systems. As a Volume Neutron Source (VNS) for materials testing, a steady state plasma is particularly desirable for obtaining the required neutron fluence [1]. For a small, steady state tokamak to be a viable candidate for a VNS, it requires replacing the inductively driven current with non-inductive sources. This requires taking maximum advantage of the bootstrap current driven by the density and temperature gradients in the plasma to ease the burden on the noninductive current drive sources. The recently observed Enhanced Reverse Shear (ERS) plasmas in the Tokamak Fusion Test Reactor (TFTR) [2] and Doublet III-D (DIII-D) [3] have been shown to yield enhanced MHD stability and confinement in the core. This behavior has been predicted by theoretical studies $[4,5]$ which also showed that such plasmas have better alignment of the bootstrap with the current profiles required to attain the improved configuration.

We examine the bootstrap current for two reference
Table I VNS Parameters

\begin{tabular}{lcc}
\hline Parameter & VNS-SC & VNS-ST \\
\hline$R_{0}(\mathrm{~m})$ & 4.11 & 0.79 \\
$a_{0}(\mathrm{~m})$ & 1.02 & 0.60 \\
$A$ & 4.04 & 1.31 \\
$\kappa$ & 2.1 & 2.3 \\
$\delta$ & 0.5 & 0.5 \\
$B_{\text {to }}(\mathrm{T})$ & 5.8 & 1.7 \\
$I_{\text {tot }}(\mathrm{MA})$ & 5.4 & 9.9 \\
$P_{\text {fus }}(\mathrm{MW})$ & 384 & 31 \\
$\beta_{N}$ & 4.1 & 5.9 \\
$n\left(a_{0}\right)$ & $n(0) / 3$ & $n(0) / 3$ \\
$T\left(a_{0}\right)(\mathrm{keV})$ & 0.5 & 0.5 \\
$q(0)$ & 3.5 & 3.5 \\
$q_{\min }$ & 1.5 & 1.5
\end{tabular}

small tokamak VNS designs that provide $1 \mathrm{MW} / \mathrm{m}^{2}$ of neutron wall loading, using target plasma profiles representative of ERS plasmas. The major parameters are shown in Table $I$. One is a larger aspect ratio $(A=4.04)$ design using superconducting coils (VNS-SC) and the other is a small aspect ratio $(A=1.31)$ design based on a compact tokamak [6] using a solid core with normal conducting toroidal field coils (VNS-ST). Both designs are based on physics assumptions that include confinement enhanced by a factor of 2.5 over low-mode (L-Mode) operation. The normalized plasma beta, $\beta_{N}$, is defined in Table $I$ and in discussion of the results as the average toroidal beta (using the vacuum toroidal fieid at the geometric center of the plasma) divided by the critical beta:

$$
\begin{aligned}
\beta_{N} & \equiv \frac{\langle\beta\rangle_{T 0}}{\beta_{\text {crit }}}, \\
\langle\beta\rangle_{T 0}(\%) & \equiv 100 \frac{\langle p\rangle}{B_{T 0} / 2 \mu_{0}}, \\
\beta_{\text {crit }}(\%) & \equiv \frac{I_{\text {tot }}(\mathrm{MA})}{a_{0} B_{T 0}},
\end{aligned}
$$

where $p$ is the plasma kinetic pressure and units are in mks unless otherwise noted. Because of its low aspect ratio, the VNS-ST has a significant parametric contribution to the toroidal field from the toroidal current such that $\beta_{N}$ defined in terms of the total toroidal is reduced from 5.9 to 2.9 . 
Safety Factor, ERS Mode

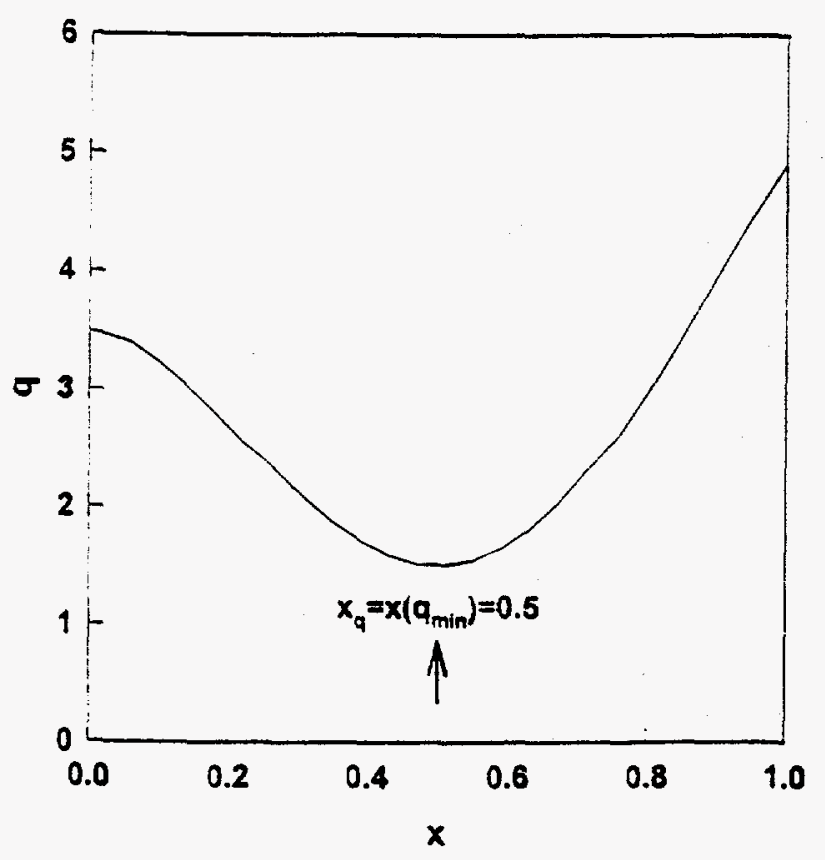

Fig. 1 The safety factor profile used to calculate the MHD equilibria for AT operation starts at 3.5 at the origin and drops to 1.5 at $x_{q}$, which is varied from $0.4-0.6$ in the sensitivity study.

\section{Normatized Plasma Profiles}

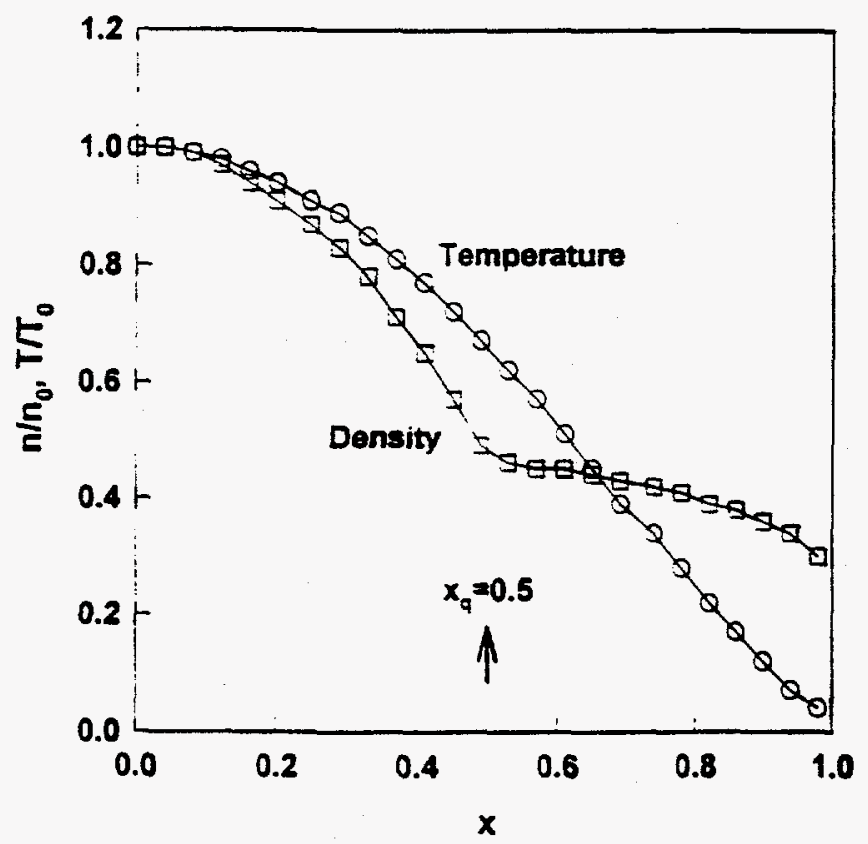

Fig. 2 The density profile shape includes a centraily peaked compo nent for $x \leq x_{q}$ to simulate ERS operation with beam heating and fueling.
Bootstrap Current: $n, T$ Variation

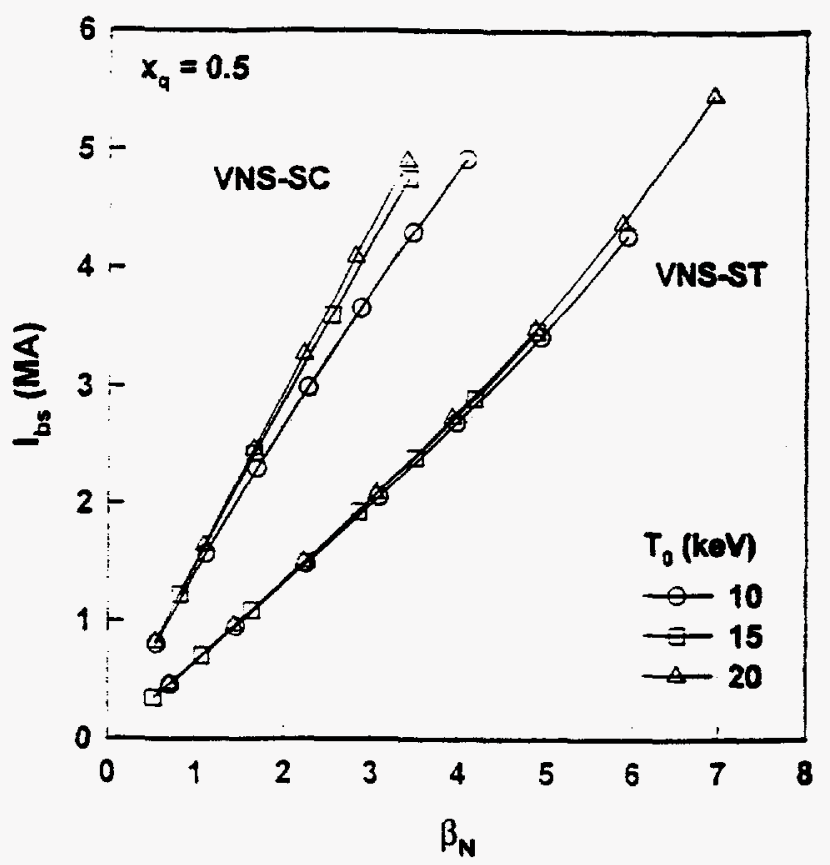

Fig. 3 The total bootstrap current is a nearly linear function of plasma beta $\left(\beta_{N} \equiv<\beta>_{T 0} / \beta_{\text {crit }}\right)$ when the shapes of the density and temperature profiles are fixed and the amplitudes varied.

\section{PLASMA MODEL}

The plasma safety factor is assumed to have reversed magnetic shear in the core as shown in Fig. 1, using a functional form $q(x)=q(0)+a_{2} x^{2}+a_{3} x^{3}+a_{4} x^{4}$, where $x=\rho / a_{0}, \rho^{2}=\sqrt{\Phi / \Phi\left(a_{0}\right)}$ and $\Phi$ is the toroidal flux enclosed by a given magnetic surface. The coefficients $a_{i}$ are adjusted iteratively with the MHD equilibrium calculation to meet three constraints on the safety factor: a local minimum value, $q_{\min }$, at the location $x_{\min }$ and a value at the edge that yields the correct total plasma current.

The plasms is consists of equal components of deuterium and tritium with equal electron and ion temperatures for this study. The profiles are taken as (Fig. 2):

$$
\begin{aligned}
T(x)= & {\left[T_{0}-T\left(a_{0}\right)\right]\left(1-x^{2}\right)^{3 / 2}+T\left(a_{0}\right), } \\
n(x)= & {\left[n_{0}-n\left(a_{0}\right)\right]\left(1-x^{2}\right)^{1 / 2}+n\left(a_{0}\right) } \\
& +n_{0}\left[1-x\left(<x_{\min }\right)^{2} / x_{\min }^{2}\right],
\end{aligned}
$$

where the parabolic term in the density profile is added for for $x<x_{\min }$ to represent the peaking observed in ERS plasmas in TFTR [2], a consequence of the improved confinement and central beam fueling. The additional density peaking factor is assumed to be a factor of two in our calculations. Strong temperature peaking is also observed in ERS plasmas, and included here with a $3 / 2$ exponent on the parabolic profile.

The bootstrap current is calculated with the recently developed NCLASS code, which performs a full matrix calculation of the parallel force and heat balance equations to 


\section{Bootstrap Fraction: $n, T$ Variation}

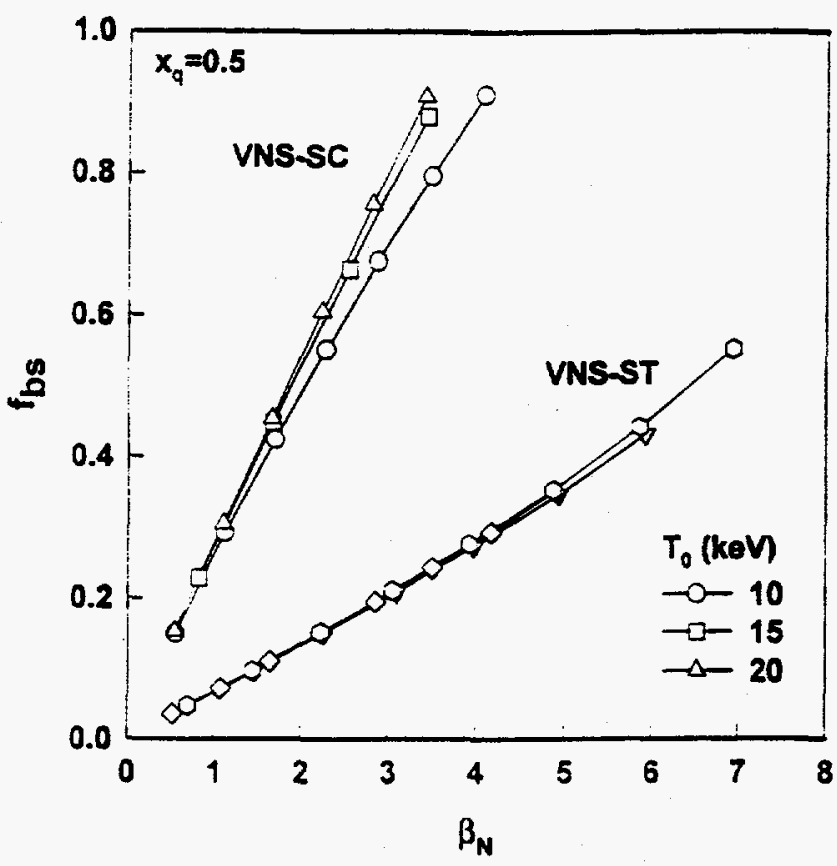

Fig. 4 The bootstrap current fraction in the VNS-SC plasma reaches unity for $\beta_{N} \approx 3.5-4.5$, and is about four times that in the VNS-SC plasma at a given $\beta_{N}$.

\section{Bootstrap Current: $x_{q}$ Variation}

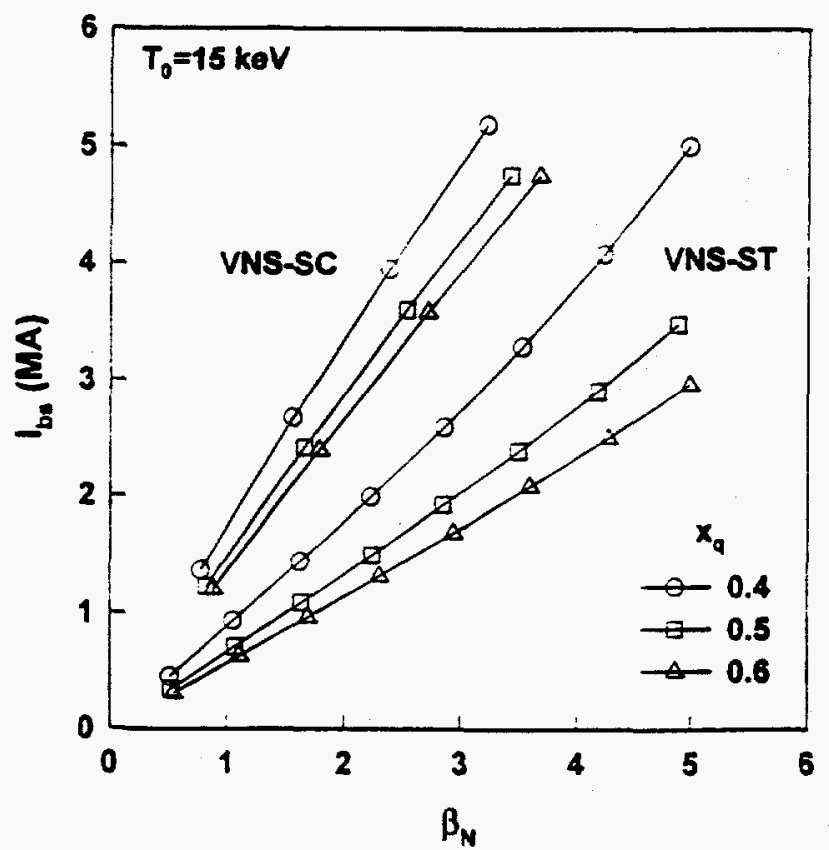

Fig. 5 As $q_{\min }$ is pushed to smaller radii the bootstrap current increases. The bootstrap current in the VNS-ST plasma shows a greater sensitivity to the position of $q_{\min }$ than it does in the VNSSC plasma.

\section{Bootstrap Fraction: $x_{q}$ Variation}

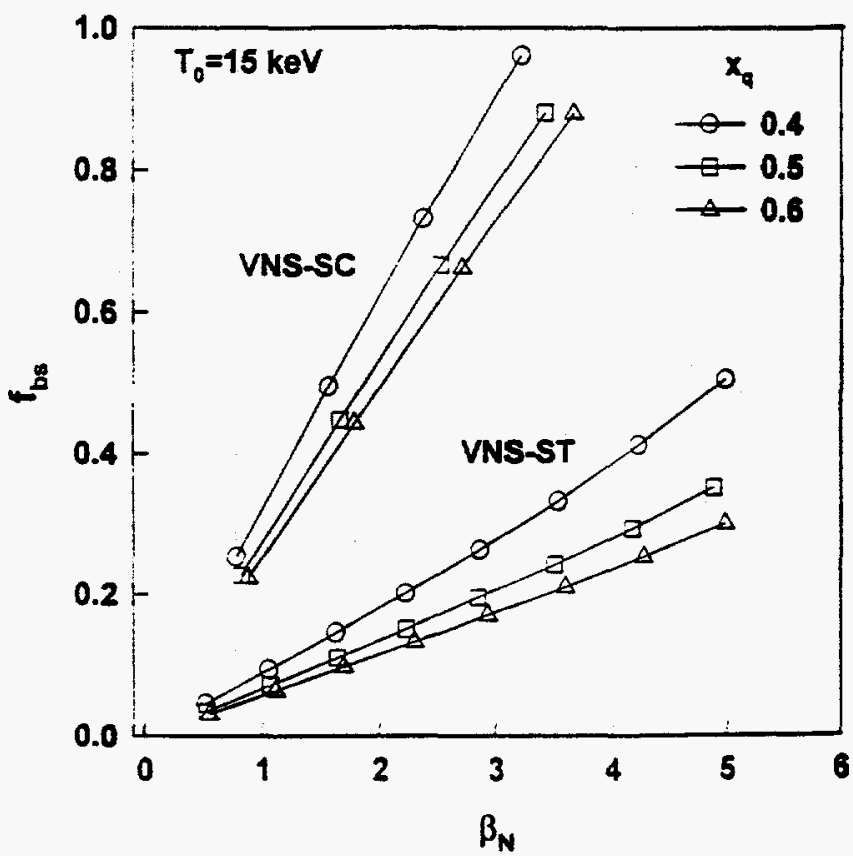

Fig. 6 The bootstrap current fraction reaches about $50 \%$ in the VNS-ST plasma at $\beta_{N}=5$.

determine the neoclassical flows and bootstrap current [7]. A new formulation of the viscosity is used that is valid at all aspect ratios and collisionalities for multiple species plasmas and finite beta equilibria [8].

\section{VNS APPLICATIONS}

Fig. 3 shows the bootstrap current as a function of $\beta_{N}$ with central temperatures of 10,15 and $20 \mathrm{keV}$. The variation with temperature and density is a result of finite collisionality; with fixed profile shapes the bootstrap current would be constant for a given plasma beta as the magnitudes of the density and temperature are varied. The larger aspect ratio VNS-SC plasma has about twice the bootstrap current as the VNS-ST plasma at a given $\beta_{N}$. Because of the higher plasma current in the VNS-ST, the bootstrap fraction is even lower at a given $\beta_{N}$ as shown in Fig. 4. However, if the diamagnetic contribution to the toroidal field from the high current is used in the definition of the average beta, the $\beta_{N}$ values for the VNS-ST are about half the values shown [i.e., $\langle\beta\rangle_{T} \approx\langle\beta\rangle_{\text {To }} / 2$ ].

If the extent of the ERS region is varied by varying $x_{\min }$, and the rise in the density profile moves with $x_{\min }$, we obtain the resuits shown in Figs 5 and 6 . This illustrates that the total bootstrap current is sensitive to the plasma profile shapes $(n, T$ and $q$ ), with the VNS-ST design showing a greater sensitivity. What is surprising at first, is that the total bootstrap current increases as the minimum in the $q$ profile is moved inward, because the plasma cross 


\section{Integrated Current}

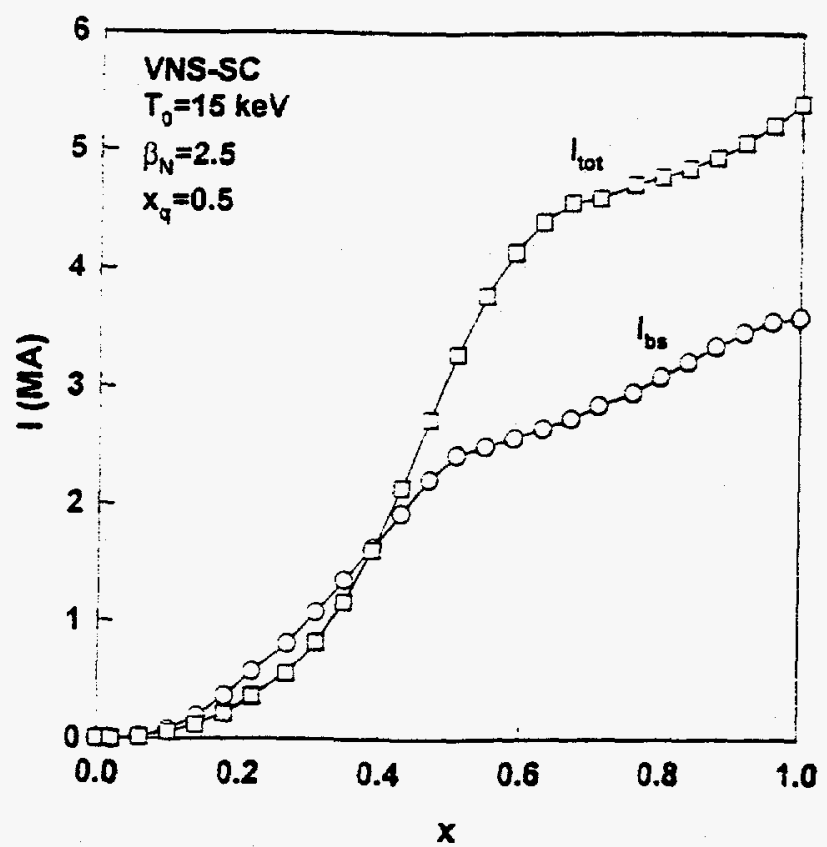

Fig. 7 The bootstrap current matches the total current at $x \approx 0.4$ in this VNS-SC case when the total bootstrap fraction is about $70 \%$ ( $I_{b a} / I_{\text {tot }}$ at the plasma edge).

\section{Bootstrap Current Density}

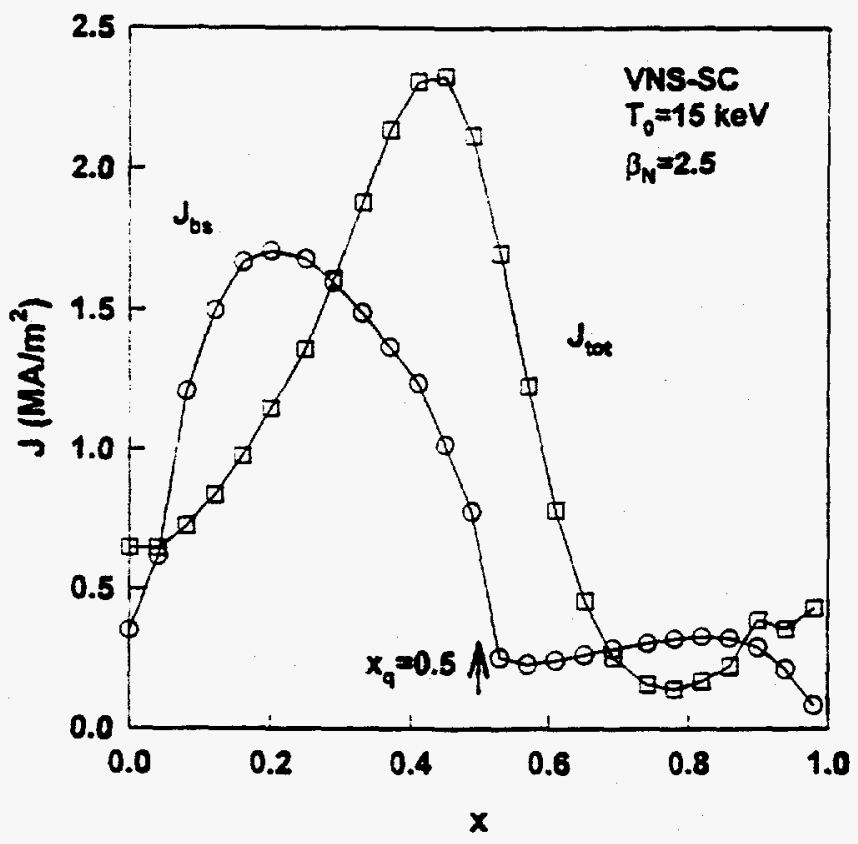

Fig. 8 The bootstrap current density in this VNS-SC case exceeds the current density required to maintain the target $q$ pronle for $x \leq 0.3$.

\section{Bootstrap Current Components}

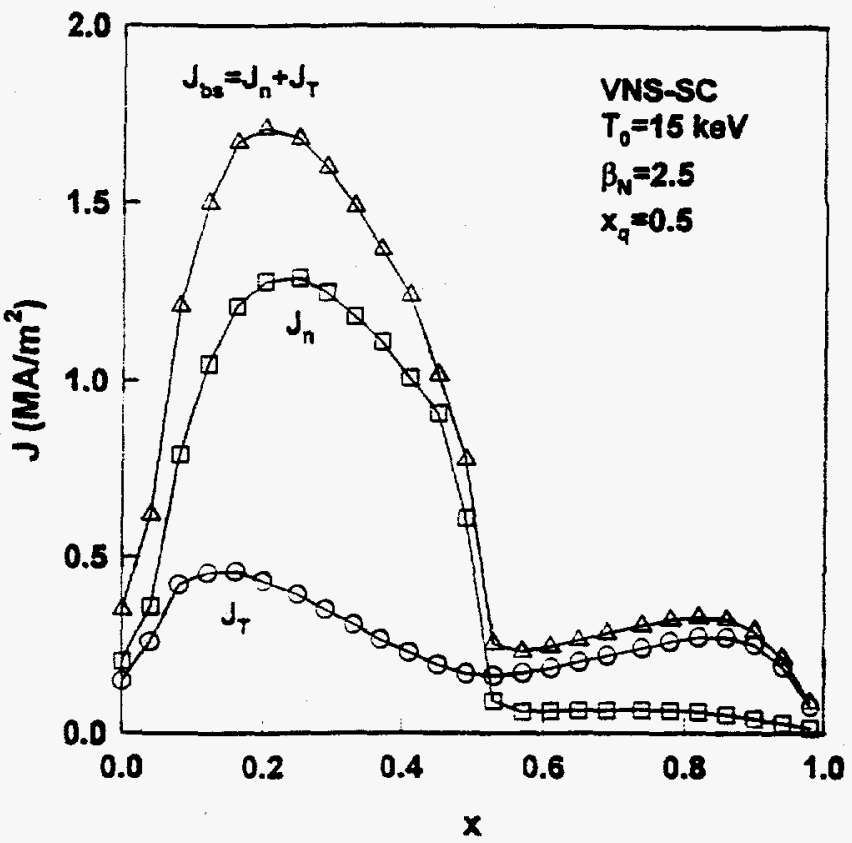

Fig. 9 The density gradient inside $x_{q}$ is primarily responsible for the enhanced bootstrap current density in the core, while the temperature gradient contribution is dominant for $x>x_{q}$.

sectional area that contains the steeper density gradient is reduced.

Further examination of the details of the bootstrap current density profile and the factors contributing to it provides an explanation for this behavior. In Fig. 7, the integrated total current and bootstrap current are shown for one of the VNS-SC cases with $70 \%$ bootstrap current fraction. The bootstrap current matches the total current at $x=0.4$, and is slightly higher than the total current for $x<0.4$. There is a reasonable agreement (in both the shape and amplitude) between bootstrap current and total current for $x<0.4$, which would allow the bootstrap current alone to generate a $q$ profile similar to the target profile if it is allowed to relax in a time dependent simulation.

The current density profiles for the VNS-SC case with $70 \%$ bootstrap current are shown in Fig. 8 , where

$J_{\alpha} \equiv \frac{\left\langle\overrightarrow{J_{\alpha}} \cdot \vec{B}\right\rangle}{B_{T 0}}$

where the subscript $\alpha$ designates either the bootstrap or total current density. On axis the bootstrap current density vanishes but is non-zero in the figure because it is averaged over a finite sized computational cell. This indicates a need for 8 small axial seed current to maintain finite $q(0)$ unless finite orbit, MHD or other effects eliminate this need. Otherwise, the bootstrap component moderately exceeds the current density required to maintain the target $q$ profile for $x \leq 0.3$, indicating no additional need for 
VNS-SC Bootstrap Amplitude Factors

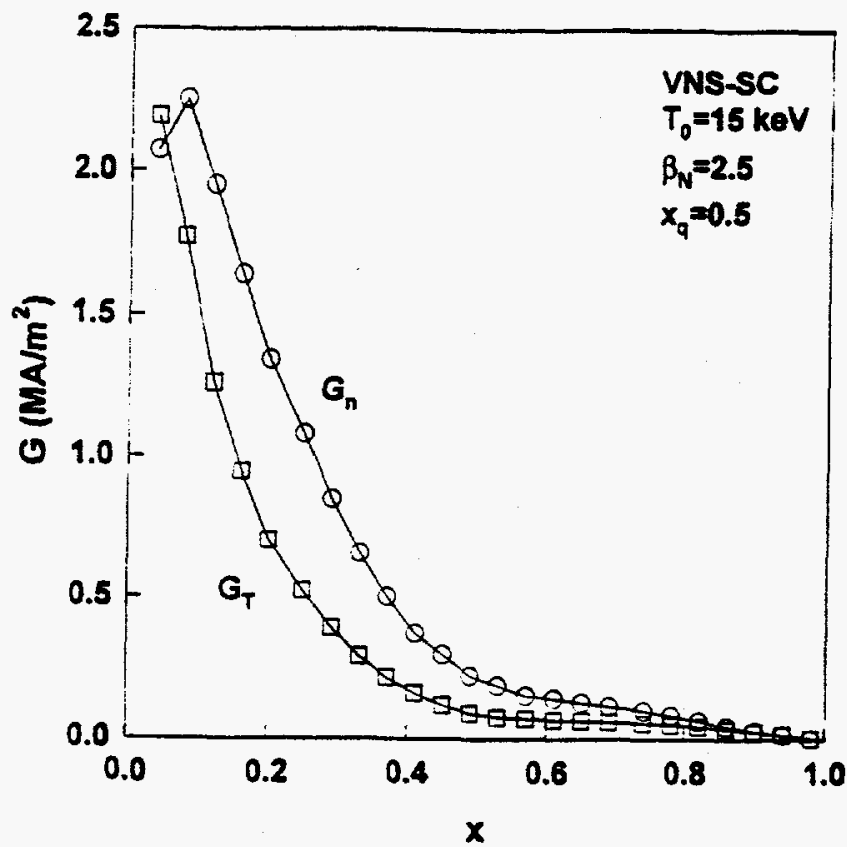

Fig. 10 The coefficients of the density and temperature gradients (bootstrap current amplitude factors $G_{n}$ and $G_{T}$, respectiveiy) that determine the bootstrap current density typically increase dramatically toward the plasma center. This indicates that gradients in either density or temperature are very efficient sources of bootstrap current in the core.

\section{VNS-ST Bootstrap Amplitude Factors}

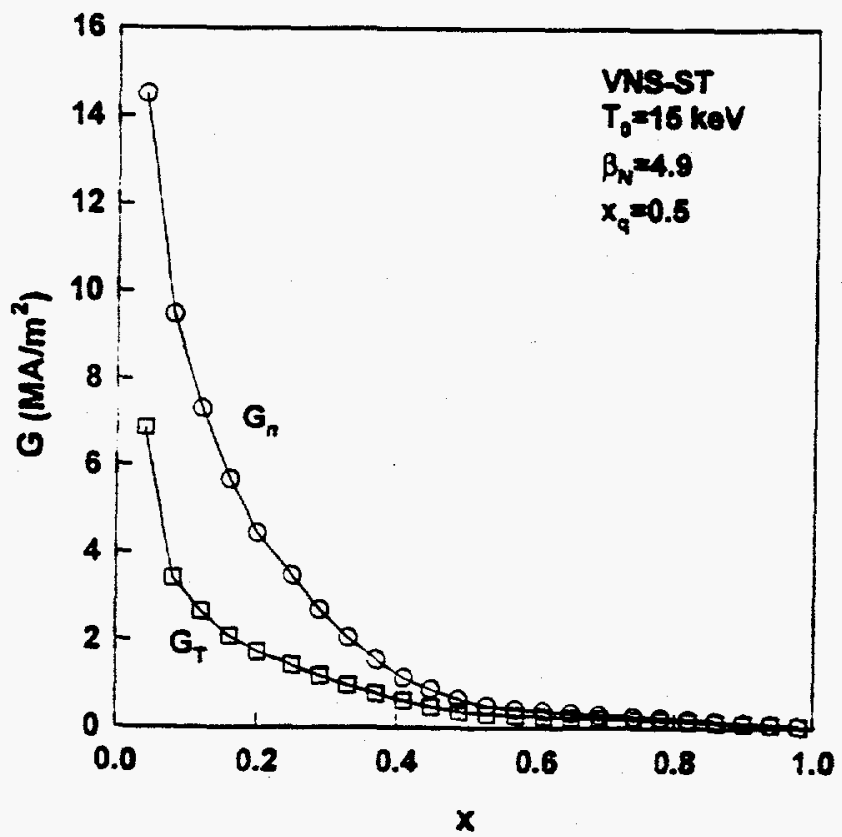

Fig. 11 The bootstrap current amplitude factors for the the smaller ratio VNS-ST plasma are both larger and more concentrated in the center than the VNSSC plasma.

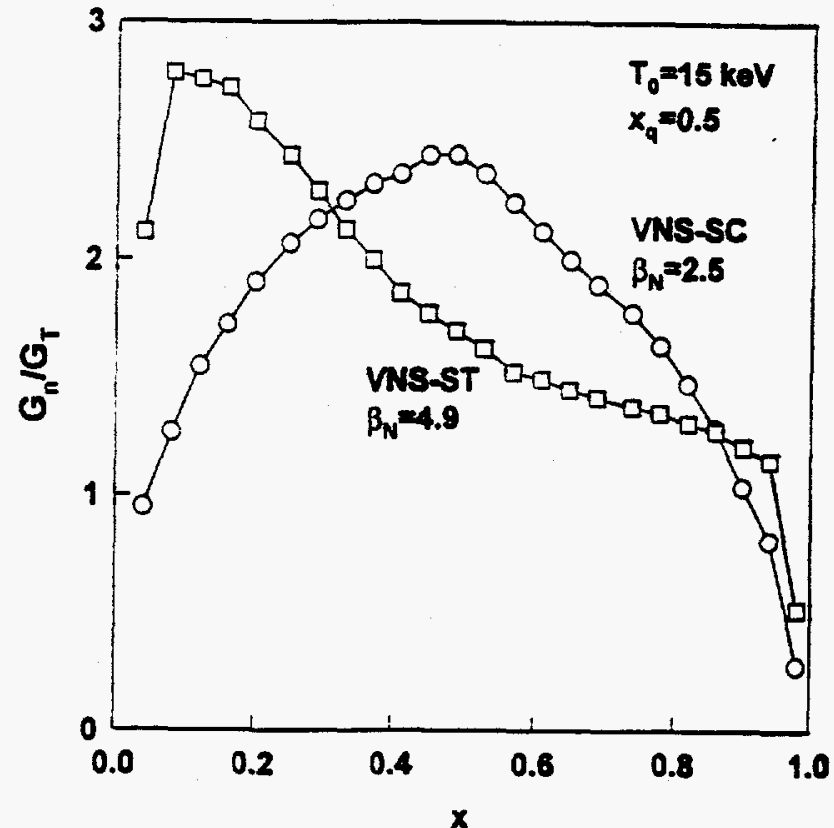

Fig. 12 A density gradient is more effective than a temperature gradient in driving bootstrap current over most of the plasma. It is a strong function of aspect ratio, MHD equilibrium and collisionality effects.

central current drive. The peak in the total current density required to maintain the position of $x_{q}$ always lies outside the peak in the bootstrap current density; this situation can only be sustained with a current drive source approximately localized to the region $x_{q}-0.1<x<x_{q}+0.1$ to pin the location of $q_{\min }$ at $x_{q}$.

A breakdown of the bootstrap current density into components driven by the particle density and temperature gradients is shown in Fig. 9. Inside $x_{q}$ the density gradient driven component is dominant, while outside this region the temperature gradient component is larger. The dominance of the density gradient component in the core is due to two factors. The density gradient associated with the assumed rapid increase in density inside $x_{q}$ has been assumed to be stronger than the temperature gradient, but a density gradient is also more effective than a temperature gradient in driving the bootstrap current.

To examine the relative effectness of the density and temperature gradients in generating bootstrap current, we normalize the components to the respective gradients of the electrons to obtain the amplitude factors shown in Fig. 10 for the VNS-SC case:

$$
\begin{aligned}
J_{b s} & =J_{n}+J_{T} \\
& =G_{n}\left|\frac{1}{n_{e}} \frac{d n_{e}}{d x}\right|+G_{T}\left|\frac{1}{T_{e}} \frac{d T_{e}}{d x}\right|
\end{aligned}
$$

These amplitude factors increase dramatically toward the axis because of increasing aspect ratio and rising $q\left(J_{b s} \propto\right.$ 
$q)$, explaining why pushing the position of $x_{q}$ and the density gradient inward enhances the total bootstrap current. In the smaller aspect ratio VNS-ST the amplitude factors are both more narrowly peaked and of higher amplitude in the center as seen in Fig. 11. This explains the greater sensitivity of the total bootstrap current in the VNS-ST plasma to the position of $q_{\min }$ as illustrated in Figs 5 and 6.

The ratio of the amplitude factors shown in Fig. 12 demonstrates that the density gradient is more effective than the temperature gradient in driving bootstrap current essentially everywhere except at the very plasma edge in both designs. Although the profiles of the drive ratio can vary because of aspect ratio and MHD equilibrium effects, they clearly show that the generation of a density gradient deeper in the plasma is the most efficient way to generate bootstrap current.

\section{SUMMARY}

Experimental observations of ERS plasmas [2, 3] have opened up a promising approach to designing steady state tokamaks with improved performance characteristics. These characteristics include enhanced confinement. MHD stability, and better alignment between the internally generated bootstrap current and the current profile required to provide access to the enhanced confinement regime. This is demonstrated in the present study of tokamak based VNS designs as well as eariier studies $[4,5]$.

Higher $q$ in the core of ERS plasmes, in combination with peaked plasma density and temperature profiles, is a very effective means of moving the peak in the bootstrap current toward the plasma center and obtaining better current alignment. This reduces the external current drive requirements to a very small axial seed current and a localized current source to pin the location of the minimum in the $q$ profile. Very high bootstrap current fractions can be attained in the VNS-SC design with normalized betas of $\beta_{N} \leq 3.5$. The bootstrap current is lower in the VNSST design because of its lower aspect ratio; the highest bootstrap fraction found in these limited cases is $50 \%$ at $\beta_{N}=5$.

The work presented in this study have focussed on one aspect of the design and operation of a VNS - the key parameters that must be considered in optimizing the bootstrap current. Many other physics issues must be addressed before chosing an optimal design. These include evaluating the capability of various noninductive current sources to provide the required amplitude and localization of the balance of the current requirements, time evolution of the current profiles to self-consistent solutions, MHD stability limits, and evolution of the density and temperature profiles to solutions that are consistent with both sources and transport properties. These are challenges the physics program is addressing in its study of advanced tokamak operting regimes.

\section{ACKNOWLEDGEMENTS}

This research was sponsored by the Office of Fusion Energy, U.S. Department of Energy, under contract DEAC05-84OR21400 with Lockheed-Martin Energy Systems, Inc.

\section{REFERENCES}

[1] M.A. Abdou, "A volumetric neutron source for fusion nuclear technology testiag and development," Fusion Eng. Des., 27. 111-153, 1995.

[2] F.M. Ievinton, M.C. Zarnstorf, S.H. Batha, M. Bell, R.E. Beil. RV. Budny, C. Bush, E. Fredrickson. A. Janos, J.Manicicam, A. Ramsey, G.L. Schmidt, E. Synakowaki, G. Taylor, "Improved confinement with reversed magnetic shear in TFTR, "unpublished.

[3] E.J. Strait, et al., unpublished.

[4] C. Kessed, J. Manicleam, G. Rewoldt, W.M. Tang, "Improved plasma performance in tolamaks with negative magnetic shear," Phys. Rev. Lett., 72, 1212-1215, 1994.

[5] A.D. Turnbull, T.S. Taylor, Y.R. Lin-Liu, H. St John, "High beta and enhanced confinement in a second stable core VHmode advanced tokamak," Phys. Rev. Lett., 74, 718-721, 1995.

[6] Y-K.M. Peng, R.J. Colchin, C.L. Hedrick. J.D. Gelambos, J. Sheffield, D.J. Strickler, R.A. Blanken. A. Syiken, D.C. Robinson, A.W. Morris, J. Hugill, T.C. Hender, S.K. Erente, H.R. Wilson. "Physics progresa towards compact tokamak reao. tors with normal conducting toroidal feld coils," in Plasma Physics and Controlled Nuclear Fusion Research, (Proc. 13th Int. Conf. Seville, 1994), IAEA, Vienna in press.

[7] S.P. Hirahman, D.J. Sigmar, "Neoclasaical transport of impurities in tolamak plasmas," Nucl. Fusion, 21, 1079-1201, 1981.

[8] K.C. Shaing, M. Yolasyama, M. Wakatan, C.T. Hsu, "An approximate analytic expression for plasma viscosity in finite aspect ratio tolamaks and its applications," Phys. Plasman, in prose.

\section{DISCLAIMER}

This report was prepared as an account of work sponsored by an agency of the United States Government. Neither the United States Government nor any agency thereof, nor any of their employees, makes any warranty, express or implied, or assumes any legal liability or responsibility for the accuracy, completeness, or usefulness of any information, apparatus, product, or process disclosed, or represents that its use would not infringe privately owned rights. Reference herein to any specific commercial product, process, or service by trade name, trademark, manufacturer, or otherwise does not necessarily constitute or imply its endorsement, recommendation, or favoring by the United States Government or any agency thereof. The views and opinions of authors expressed herein do not necessarily state or reflect those of the United States Government or any agency thereof. 